Proyecciones Journal of Mathematics

Vol. 29, $\mathrm{N}^{\circ}$ 1, pp. 75-82, May 2010.

Universidad Católica del Norte

Antofagasta - Chile

\title{
BOUNDEDNESS AND UNIFORM CONVERGENCE IN B-DUALS
}

\author{
CHARLES SWARTZ \\ NEW MEXICO STATE UNIVERSITY, U.S.A. \\ Received : November 2009. Accepted : December 2010
}

\begin{abstract}
Suppose $E$ is a vector valued sequence space with operator valued $\beta$-dual $E^{\beta Y}$. If the space $E$ satisfies certain gliding hump conditions, we consider the connection between pointwise bounded subsets $A$ of $E^{\beta Y}$ and the uniform convergence of the elements of $A$. For linear operators our results contain results of Li, Wang and Zhong for the spaces $c_{0}(X)$ and $l^{p}(X)$.
\end{abstract}


In this paper we consider the connection between boundedness and uniform convergence for subsets in the $\beta$-dual of a vector valued sequence space. In particular, we consider pointwise bounded subsets in $\beta$-duals and uniform convergence of the pointwise bounded subsets over subsets of the sequence space. Our results hold for vector valued sequence spaces which satisfy certain gliding hump properties and contain results of $\mathrm{Li}$, Wang and Zhong ([LWZ]) for linear operators and the sequence spaces $c_{0}(X)$ and $l^{p}(X)$ as special cases.

We begin by fixing the notation and terminology. Let $X, Y$ be Hausdorff topological vector spaces and let $E$ be a vector space of $X$ valued sequences containing the space $c_{00}(X)$ of all $X$ valued sequences which are eventually 0 . The $\beta$-dual of $E$ with respect to $Y, E^{\beta Y}$, is defined to be

$$
E^{\beta Y}=\left\{\left\{T_{j}\right\} \subset L(X, Y): \sum_{j=1}^{\infty} T_{j} x_{j} \text { converges for every } x=\left\{x_{j}\right\} \in E\right\},
$$

where $L(X, Y)$ is the space of continuous linear operators from $X$ into $Y$. If $T=\left\{T_{j}\right\} \in E^{\beta Y}$ and $x=\left\{x_{j}\right\} \in E$, we write $T \cdot x=\sum_{j=1}^{\infty} T_{j} x_{j}$.

We now describe 2 gliding hump properties which will be used in the sequel. An interval in $\mathbf{N}$ is a set of the form $[m, n]=\{j \in \mathbf{N}: m \leq j \leq n\}$, where $m \leq n$, and a sequence of intervals, $\left\{I_{j}\right\}$, is increasing if $\max I_{j}<$ $\min I_{j+1}$. If $I \subset \mathbf{N}, \chi_{I}$ will denote the characteristic function of $I$ and if $x=\left\{x_{j}\right\}$ is any scalar or vector valued sequence, $\chi_{I} x$ will denote the coordinatewise product of $\chi_{I}$ and $x$.

Throughout let $\mathcal{F}$ be a family of subsets of $E$.

Definition 1. The space $E$ has the signed $\mathcal{F}$ gliding hump property (signed $\mathcal{F}$-GHP) if for every $F \in \mathcal{F}$ whenever $\left\{x^{j}\right\} \subset F$ and $\left\{I_{j}\right\}$ is an increasing sequence of intervals, there exist a sequence of signs $\left\{s_{j}\right\}$ and a subsequence $\left\{n_{j}\right\}$ such that the coordinate sum of the series $\sum_{j=1}^{\infty} s_{j} \chi_{I_{n_{j}}} x^{n_{j}}$ belongs to $E$. If all signs $s_{j}$ can be chosen equal to 1 , then $E$ has the $\mathcal{F}$ gliding hump property $(\mathcal{F}-G H P)$.

Examples of spaces and families with signed $\mathcal{F}$-GHP are given in [Sw4]. In particular, if $\mathcal{F}$ is the family of finite sets, signed $\mathcal{F}$-GHP is just the signed weak gliding hump property and if $\mathcal{F}$ is the family of bounded subsets of a K-space $E$, signed $\mathcal{F}$-GHP is the signed strong gliding hump property. Further examples are given in [Sw4] and another example is given later.

Definition 2. The space $E$ has the infinite gliding hump property $(\infty$ GHP) if whenever $x \in E$ and $\left\{I_{j}\right\}$ is an increasing sequence of intervals, 
there exist a subsequence $\left\{n_{j}\right\}$ and $a_{n_{j}}>0, a_{n_{j}} \rightarrow \infty$ such that every subsequence of $\left\{n_{j}\right\}$ has a further subsequence $\left\{p_{j}\right\}$ such that the coordinate sum of the series $\sum_{j=1}^{\infty} a_{p_{j}} \chi_{I_{p_{j}}} x$ belongs to $E$.

The $\infty-G H P$ was introduced in [Sw2] to treat Orlicz-Pettis Theorems for multiplier convergent series with respect to the strong topology.

Examples of spaces with $\infty$-GHP are given in Appendices $\mathrm{B}$ and $\mathrm{C}$ of [Sw3]. Another example is given later.

We now state a lemma which will be used. The proof is as in the proof of Lemma 10 of [Sw4] or Lemma 2.15 of [Sw3].

Lemma 3. Let $A \subset E^{\beta Y}$ and $B \subset F$. If the series $\sum_{j=1}^{\infty} T_{j} x_{j}$ do not converge uniformly for $T \in A$ and $x \in F$, then there exist a balanced neighborhood of $0, V$, in $Y,\left\{T^{k}\right\} \subset A,\left\{x^{k}\right\} \subset F$ and an increasing sequence of intervals $\left\{I_{k}\right\}$ such that $\sum_{l \in I_{k}} T_{l}^{k} x_{l}^{k} \notin V$.

We now prove our major result. If $z \in X$ and $j \in \mathbf{N}$, let $e^{j} \otimes z$ be the sequence with $z$ in the $j^{\text {th }}$ coordinate and 0 in the other coordinates.

Theorem 4. Assume $E$ has $\infty$-GHP and $\mathcal{F}$-GHP. If $A \subset E^{\beta Y}$ is pointwise bounded on $E$ (i.e., $\{T \cdot x: T \in A\}$ is bounded for each $x \in E$ ) and $F \in \mathcal{F}$, then the series $\sum_{j=1}^{\infty} T_{j} x_{j}$ converge uniformly for $T \in A$ and $x \in F$.

Proof: Suppose the conclusion fails and let the notation be as in the lemma so

$$
\text { (*) } \sum_{l \in I_{k}} T_{l}^{k} x_{l}^{k} \notin V .
$$

By $\mathcal{F}$-GHP, there exist signs $\left\{s_{j}\right\}$ and a subsequence $\left\{n_{j}\right\}$ such that

$$
\sum_{j=1}^{\infty} \sum_{l \in I_{n_{j}}} s_{j} e^{l} \otimes x_{l}^{n_{j}}=x \in E
$$

. To avoid cumbersome notation later assume that $n_{j}=j$ so

$$
(* *) \sum_{l \in I_{j}} T_{l}^{j} x_{l}=s_{j} \sum_{l \in I_{j}} T_{l}^{j} x_{l}^{j} \notin V .
$$

Now apply $\infty$-GHP to $x$ and let the notation be as in Definition 2. Define a matrix

$$
M=\left[\sum_{l \in I_{n_{j}}}\left(\frac{1}{a_{n_{i}}} T_{l}^{n_{i}}\right)\left(a_{n_{j}} x_{l}\right)\right]=\left[m_{i j}\right] .
$$


We now use the Antosik-Mikusinski Matrix Theorem ([Sw4]Appendix D, [Sw1]2.2.4) to show the diagonal of $M$ converges to 0 ; this will contradict $(* *)$.

First, the columns of $M$ converge to 0 by the pointwise boundedness of $A$ and the fact that $a_{n_{i}} \rightarrow \infty$. Next, given any subsequence of $\left\{n_{j}\right\}$ there exists a further subsequence such that

$$
\sum_{j=1}^{\infty} a_{p_{j}} \chi_{I_{p_{j}}} x=\sum_{j=1}^{\infty} a_{p_{j}} \sum_{l \in I_{p_{j}}} e^{l} \otimes x_{l}=y \in E .
$$

Then

$$
\sum_{j=1}^{\infty} m_{i p_{j}}=\sum_{j=1}^{\infty} \sum_{l \in I_{p_{j}}}\left(\frac{1}{a_{n_{i}}} T_{l}^{n_{i}}\right)\left(a_{p_{j}} x_{l}\right)=\left(\frac{1}{a_{n_{i}}} T_{l}^{n_{i}}\right) \cdot y \rightarrow 0
$$

by pointwise boundedness. By the Antosik-Mikusinski Matrix Theorem the diagonal of $M$ goes to 0 .

The special case of Theorem 4 for spaces with the signed weak gliding hump property is given in [Sw3] 2.32.

Example 5. Without the $\infty$-GHP the conclusion of Theorem 4 may fail. Let $E=l^{\infty}$ so $E^{\beta}=l^{1}$. Then $\left\{e^{i}: i \in \mathbf{N}\right\} \subset l^{1}$ is pointwise bounded on $l^{\infty}$, but if $e$ is the constant sequence $\{1\}$ the series $\sum_{i} e^{i} \cdot e$ do not converge uniformly in $i$.

We next consider conditions which insure pointwise boundedness in $\beta$ duals. For this we need a preliminary observation.

Proposition 6. If $A \subset E^{\beta Y}$ is pointwise bounded on $E$, then $\left\{T_{j} x\right.$ : $\left.\left\{T_{j}\right\} \in A\right\}$ is bounded in $Y$ for every $x \in X$ and $j \in \mathbf{N}$.

Proof: Note $e^{j} \otimes x \in E$ for $x \in X, j \in \mathbf{N}$ and $T \cdot e^{j} \otimes x=T_{j} x$.

We now consider the converse of Theorem 4 .

Theorem 7. If $A \subset E^{\beta Y}$ is such that $\left\{T_{j} x:\left\{T_{j}\right\} \in A\right\}$ is bounded in $Y$ for every $x \in X$ and $j \in \mathbf{N}$ and for every $x \in E$ the series $\sum_{j=1}^{\infty} T_{j} x_{j}$ converge uniformly for $T \in A$, then $A$ is pointwise bounded on $E$. 
Proof: Let $x \in E$ and $U$ be a neighborhood of 0 in $Y$. Let $V$ be a balanced neighborhood of 0 in $Y$ such that $V+V \subset U$. There exists $N$ such that $\sum_{j=n}^{\infty} T_{j} x_{j} \in V$ for $n \geq N, T \in A$. Let $\left\{T^{k}\right\} \subset A$. We show $\lim \left(T^{k} \cdot x\right) / k=0$. We have $\lim \frac{1}{k} \sum_{j=1}^{N} T_{j}^{k} x_{j}=0$ by hypothesis so there exists $k_{0}$ such that $\frac{1}{k} \sum_{j=1}^{N} T_{j}^{k} x_{j} \in V$ for $k \geq k_{0}$. Therefore, if $k \geq k_{0}$, then

$$
\frac{1}{k} \sum_{j=1}^{\infty} T_{j}^{k} x_{j}=\frac{1}{k} \sum_{j=1}^{N} T_{j}^{k} x_{j}+\frac{1}{k} \sum_{j=N+1}^{\infty} T_{j}^{k} x_{j} \in V+V \subset U
$$

since $V$ is balanced.

From Theorems 4 and 7 , we have a characterization of pointwise bounded sets in $E^{\beta Y}$.

Corollary 8. Assume $E$ has $\infty-G H P$ and $\mathcal{F}-G H P$ and $A \subset E^{\beta Y}$. The following are equivalent: (1) $A$ is pointwise bounded on $E$, (2) $\left\{T_{j} x\right.$ : $\left.\left\{T_{j}\right\} \in A\right\}$ is bounded in $Y$ for every $x \in X$ and $j \in \mathbf{N}$ and for every $x \in E$ the series $\sum_{j=1}^{\infty} T_{j} x_{j}$ converge uniformly for $T \in A$, (3) $\left\{T_{j} x:\left\{T_{j}\right\} \in A\right\}$ is bounded in $Y$ for every $x \in X$ and $j \in \mathbf{N}$ and for every $x \in E, F \in \mathcal{F}$ the series $\sum_{j=1}^{\infty} T_{j} x_{j}$ converge uniformly for $T \in A, x \in F$.

From the proof of Theorem 7 we also have a sufficient condition for uniform boundedness in $E^{\beta Y}$. If $B \subset E$ and $j \in \mathbf{N}$, we write $B_{j}=\left\{x_{j}\right.$ : $\left.x=\left\{x_{j}\right\} \in B\right\}$.

Theorem 9. If $A \subset E^{\beta Y}, B \subset E$ are such that $\left\{T_{j} x:\left\{T_{j}\right\} \in A, x \in B_{j}\right\}$ is bounded in $Y$ for every $j \in \mathbf{N}$ and the series $\sum_{j=1}^{\infty} T_{j} x_{j}$ converge uniformly for $T \in A, x \in B$, then $A$ is uniformly bounded on $B$.

From Theorems 4 and 9 we have a uniform boundedness principle.

Corollary 10. Assume $E$ has $\infty-G H P$ and $\mathcal{F}$-GHP and $A \subset E^{\beta Y}$. The following are equivalent: (i) $A$ is uniformly bounded on each $F \in \mathcal{F}$, (ii) for each $F \in \mathcal{F},\left\{T_{j} x:\left\{T_{j}\right\} \in A, x \in F_{j}\right\}$ is bounded in $Y$ for every $j \in \mathbf{N}$ and the series $\sum_{j=1}^{\infty} T_{j} x_{j}$ converge uniformly for $T \in A, x \in F$.

A similar uniform boundedness principle for spaces satisfying different gliding hump assumptions has been established in Corollary 21 of [SS].

We now give examples of spaces and families to which the results above apply. We first consider the family of subsets of a sequence space $E$ with uniform tails. A sequence space $E$ is a K-space if $E$ has a vector topology such that the coordinate maps $x=\left\{x_{j}\right\} \rightarrow x_{j}$ for $E$ into $X$ are continuous for each $j \in \mathbf{N}$. 
Definition 11. If $E$ is a $K$-space, a subset $F \subset E$ has uniform tails if whenever $\left\{x^{k}\right\} \subset F$, then there exists $\left\{n_{k}\right\}$ such that $\lim _{N \rightarrow \infty} \sum_{j=N}^{\infty} e^{j} \otimes$ $x_{j}^{n_{k}}=0$ uniformly for $k \in \mathbf{N}$, where $\sum_{j=N}^{\infty} e^{j} \otimes x_{j}^{n_{k}}$ is the coordinatewise sum of the series.

Sets with uniform tails were introduced in [Sw4]. It was shown in Theorem 12 of [Sw4] that in some sense the family of subsets with uniform tails is optimal for the uniform convergence of a series in a $\beta$-dual. It was shown in [Sw4], Theorem 6 , that if $E$ is a complete, quasi-normed K-space, then the family of subsets $\mathcal{F}$ with uniform tails has the $\mathcal{F}$-GHP. It was also shown in [Sw4], Theorem 7, that if $E$ is a quasi-normed AK-space with a monotone basis, then the family of precompact subsets of $E$ has uniform tails. Sets with uniform tails for the special spaces $c_{0}(X)$ and $l^{p}(X)$ were considered in [LWZ] where uniform convergence in the $\beta$-duals of these spaces is considered.

In order to compare our results to those of [ZLY] where the space $c_{0}(X)$ is considered, we first consider the family of subsets of $c_{0}(X)$ with uniform tails. Here $c_{0}(X)$ is the space of all $X$ valued sequences which converge to 0 . We describe the topology of $c_{0}(X)$. Let $\mathcal{X}$ be the family of all continuous quasi-norms which generate the topology of $X$ ( the topology of any topological vector space is always generated by a family of quasi-norms $[\mathrm{BM}]$ ) and if $p \in \mathcal{X}$, set $\bar{p}(x)=\sup _{i} p\left(x_{i}\right)$ for $x=\left\{x_{i}\right\} \in c_{0}(X)$. Then the family of quasi-norms $\{\bar{p}: p \in \mathcal{X}\}$ generate the topology of $c_{0}(X)$ under which $c_{0}(X)$ is a $\mathrm{K}$-space (actually an AK-space ([Sw3], AppendixC )).

Proposition 12. If $\mathcal{F}$ is the family of all subsets of $c_{0}(X)$ with uniform tails, then $c_{0}(X)$ has $\mathcal{F}$-GHP.

Proof: Let $F \subset c_{0}(X)$ have uniform tails, $\left\{x^{j}\right\} \subset F$ and $\left\{I_{j}\right\}$ be an increasing sequence of intervals. We claim $x=\sum_{j=1}^{\infty} \chi_{I_{j}} x^{j} \in c_{0}(X)$. Let $p \in \mathcal{X}$ and $\epsilon>0$. There exists $N$ such that $n \geq N$ implies $\bar{p}\left(\sum_{l=n}^{\infty} e^{l} \otimes x_{l}^{j}\right)<$ $\epsilon$ for all $j$. Therefore, $p\left(x_{l}^{j}\right)<\epsilon$ for $j \in \mathbf{N}, l \geq N$. If $\min I_{j}>N, p\left(x_{l}^{j}\right)<\epsilon$ for $l \in I_{j}$ so $x \in c_{0}(X)$.

Next, to compare our results with those in [ZLW] for linear operators, we consider the $\infty$-GHP for $c_{0}(X)$. Recall a space $X$ is braked $([\mathrm{Kh}])$ if for every $\left\{x_{j}\right\} \in c_{0}(X)$ there is a scalar sequence $\left\{t_{j}\right\} \in c_{0}$ and a sequence $\left\{z_{j}\right\} \in$ $c_{0}(X)$ such that $\left\{x_{j}\right\}=\left\{t_{j} z_{j}\right\}$ (in the terminology of [Sw3], Appendix B.37, we would say that $c_{0}(X)$ is $c_{0}$ factorable).

Proposition 13. If $X$ is braked, then $c_{0}(X)$ has $\infty-G H P$. 
Proof: Let $x \in c_{0}(X), x=t z$ with $t \in c_{0}$ and $z \in c_{0}(X)$. Let $\left\{I_{j}\right\}$ be an increasing sequence of intervals. Set $b_{j}=\max _{i \in I_{j}}\left|t_{i}\right|$. If $b_{j}=0$ for large $j$, then $\sum_{j=1}^{\infty} j \chi_{I_{j}} x \in c_{0}(X)$ so we may assume $b_{n_{j}}>0$ for some subsequence $n_{j}$. Put $a_{n_{j}}=1 / b_{n_{j}}, v_{j}=t_{j} a_{n_{k}}$ for $j \in I_{n_{k}}$ and $v_{j}=0$ otherwise. Then $\left|t_{j} a_{n_{k}}\right| \leq 1$ if $j \in I_{n_{k}}$ so $v=\left\{v_{j}\right\} \in l^{\infty}$ and $v z \in c_{0}(X)$. Therefore,

$$
\sum_{j=1}^{\infty} e^{j} \otimes(v z)_{j}=\sum_{k=1}^{\infty} \sum_{j \in I_{n_{k}}} e^{j} \otimes a_{n_{k}} t_{j} z_{j}=\sum_{k=1}^{\infty} \sum_{j \in I_{n_{k}}} a_{n_{k}} e^{j} \otimes x_{j} \in c_{0}(X) .
$$

Since the same argument can be applied to any subsequence, the result follows.

From Corollaries 8 and 10, we then have

Corollary 14. Assume $X$ is braked and $A \subset c_{0}(X)^{\beta Y}$. The following are equivalent: (1) $A$ is pointwise bounded on $c_{0}(X)$, (2) $\left\{T_{j} x: T=\left\{T_{j}\right\} \in A\right\}$ is bounded for each $x \in X, j \in \mathbf{N}$ and the series $\sum_{j=1}^{\infty} T_{j} x_{j}$ converge uniformly for $T \in A$ and $x=\left\{x_{j}\right\}$ belonging to any subset with uniform tails.

Corollary 14 gives Theorem 3.1 of [ZLY] for the case of linear operators. It should be pointed out that the results of [ZLY] hold for quasihomogeneous operators. We have not considered this class of operators.

Corollary 15. Assume $X$ is braked and $A \subset c_{0}(X)^{\beta Y}$. The following are equivalent: (i) $A$ is uniformly bounded on sets with uniform tails, (ii) for each subset $F \subset c_{0}(X)$ with uniform tails $\left\{T_{j} x: T=\left\{T_{j}\right\} \in A, x \in F_{j}\right\}$ is bounded for $j \in \mathbf{N}$ and the series $\sum_{j=1}^{\infty} T_{j} x_{j}$ converge uniformly for $T \in A, x \in F$.

From Theorems 6 and 7 of [Sw4], we also have

Remark 16. Assume $E$ has $\infty$-GHP. If $E$ is a complete quasi-normed $K$-space [resp., $E$ is a complete quasi-normed AK-space with a monotone basis], then the 3 conditions of Corollary 8 and the 2 conditions of Corollary 10 are equivalent if $\mathcal{F}$ is the family of all subsets with uniform tails [resp., the family of all precompact subsets].

It should be pointed out that Zhong, Li and Yang in [ZLY] also addressed the problem of convergent sequences of quasi-homogeneous operators on $c_{0}(X)$ and their connection to uniform converence of series. For linear operators on sequence spaces with gliding hump properties these questions have been considered in chapters 2 and 11 of [Sw3]. 


\section{References}

[Kh] Khaleelulla, S. M., Counterexamples in Topological Vector Spaces, Springer-Verlag, Heidelberg, (1982).

[LWZ] Li, R., Wang, F., Zhong, S., The strongest intrinsic meaning of sequential-evaluation convergence, Topology and its Appl., 154, pp. 1195-1205, (2007).

[SS] Stuart, C., Swartz, C., Uniform Convergence in the Dual of a VectorValued Sequence Space, Taiwnese J. Math., 7, pp. 665-676, (2003).

[Sw1] Swartz, C., Infinite Matrices and the Gliding Hump, World Sci. Publ., (1996).

[Sw2] Swartz, C.,Orlicz-Pettis Theorems for Multiplier Convergent Operator Valued Series, Proy. J. Math., 23, pp. 61-72, (2004).

[Sw3] Swartz, C., Multiplier Convergent Series, World Sci. Publ., Singapore, (2009).

[Sw4] Swartz, C., An Abstract Gliding Hump Property, Proy.J. Math., 28, pp. 89-109, (2009).

[ZLW] Zhong, S., Li, R., Yang, H., Summability Results for Matrices of Quasi-homogeneous Operators, Proy. J. Math., 27, pp. 249-258, (2008).

\section{Charles Swartz}

Mathematics Department

New Mexico State University

Las Cruces

NM 88003

U. S. A.

e-mail: cswartz@nmsu.edu 